\title{
Influence of Gas Phase Equilibria on the Chemical Vapor Deposition of Graphene
}

\author{
Amanda M. Lewis, Brian Derby, and Ian A. Kinloch* \\ School of Materials, University of Manchester, Grosvenor Street, M13 9PL, U.K.
}

\begin{abstract}
We have investigated the influence of gas phase chemistry on the chemical vapor deposition of graphene in a hot wall reactor. A new extended parameter space for graphene growth was defined through literature review and experimentation at low pressures ( $\geq 0.001 \mathrm{mbar}$ ). The deposited films were characterized by scanning electron microscopy, Raman spectroscopy, and dark field optical microscopy, with the latter showing promise as a rapid and nondestructive characterization technique for graphene films. The equilibrium gas compositions

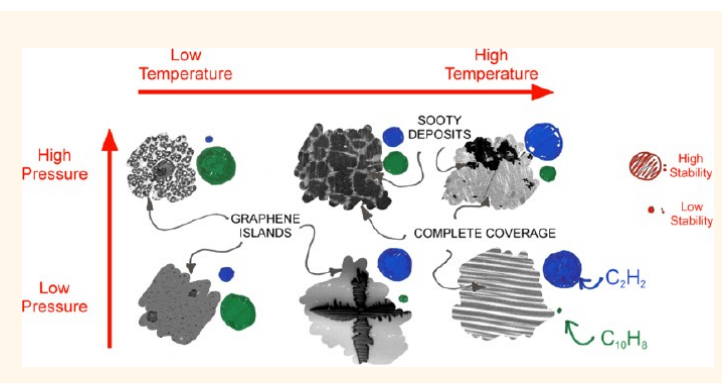
have been calculated across this parameter space. Correlations between the graphene films grown and prevalent species in the equilibrium gas phase revealed that deposition conditions associated with a high acetylene equilibrium concentration lead to good quality graphene deposition, and conditions that stabilize large hydrocarbon molecules in the gas phase result in films with multiple defects. The transition between lobed and hexagonal graphene islands was found to be linked to the concentration of the monatomic hydrogen radical, with low concentrations associated with hexagonal islands.
\end{abstract}

KEYWORDS: graphene $\cdot$ CVD $\cdot$ copper $\cdot$ equilibrium thermodynamics $\cdot$ dark field microscopy

$\mathrm{T}$ he first demonstration of graphene's ambipolar field effect in $2004^{1}$ spurred numerous studies on large-area production of this remarkable material. ${ }^{2,3}$ Early experiments focused on either the graphitization of SiC crystals leading to an epitaxial graphene coating ${ }^{4}$ or the dissolution of carbon in metal substrates at elevated temperatures followed by its segregation during cooling. ${ }^{5,6}$ However, both of these methods have difficulties; the $\mathrm{SiC}$ technique requires ultrahigh vacuums and costly precursor materials, while the segregation technique produces films with a high proportion of multilayer areas, even when cooling is carefully controlled. The chemical vapor deposition (CVD) of graphene from hydrocarbons on a copper substrate has shown the most promise to date. ${ }^{7}$ Carbon has a low solubility in copper, ${ }^{8-11}$ allowing large areas of monolayer graphene to be formed. ${ }^{12}$ Copper is etched away more readily than other metals with a low carbon solubility used for CVD of graphene, such as gold, silver, platinum, and iridium, making it practical for the transfer of deposited graphene to other surfaces.
The CVD growth process typically uses a hydrocarbon gas feedstock mixed with hydrogen flowing over a heated copper foil. The partial pressures of the hydrocarbon and the hydrogen can be further controlled through the total chamber pressure or the addition of an inert diluent gas. The copper foil is heated either directly in an unheated chamber (cold wall CVD) or by heating the entire reaction chamber volume (hot wall CVD). In a hot wall reactor, the elevated temperature of the chamber is expected to stimulate gas phase reactions, driving the gaseous composition toward thermodynamic equilibrium. ${ }^{13}$

Both the kinetics and thermodynamics of the growth process will affect the deposition of graphene and are used in classical analyses of the CVD process. ${ }^{14}$ The kinetics considers the nucleation, rate of deposition, and the mobility of carbon adspecies based on progression of chemical reactions over time, for which reaction rates and activation energies are important, and has been studied by various groups. ${ }^{15-22}$ Consideration of thermodynamic equilibria, which are time-independent and define the lowest
* Address correspondence to ian.kinloch@manchester.ac.uk.

Received for review November 9, 2012 and accepted March 12, 2013.

Published online

$10.1021 / \mathrm{nn} 305223 \mathrm{y}$

(c) XXXX American Chemical Society 
energy configuration of a system under isothermic and isobaric conditions, can also assist in determining the conditions required for graphene growth. Furthermore, the kinetic studies generally assume that the breakdown of feedstock molecules on the substrate is the initial step in the reaction pathway for graphene formation. This fundamental assumption can be corroborated only by determining that the hydrocarbon feedstock is not likely to form other molecules before colliding with the substrate through homogeneous reactions, which is the concern of thermodynamics. Despite this, the thermodynamic equilibria relevant to graphene deposition have to date been little explored. For example, Li et al. calculated the gas phase composition developed through homogeneous reactions at a pressure of 27 mbar, restricting the hydrocarbon species to a limited set. ${ }^{23}$ Here we conduct a more complete thermodynamic analysis, with the aims of collating and interpreting current results and extending the understanding of the hot wall CVD graphene process. This is achieved by reviewing the extensive graphene literature to establish a preliminary experimental parameter space for the deposition of graphene in a hot wall CVD reactor, classified by the type of coverage. This parameter space is defined using the variables of reaction temperature $\left(T_{\mathrm{r}}\right)$, total hydrogen and hydrocarbon partial pressure (the active species partial pressure, $\left.P_{\mathrm{A}}\right)$, and $\mathrm{C}: \mathrm{H}$ ratio $\left(R_{\mathrm{CH}}\right)$, with most of the data in the literature carried out at $P_{\mathrm{A}}>0.1$ mbar (10 Pa) and $T_{\mathrm{r}}>700^{\circ} \mathrm{C}$. We then experimentally extend the parameter space to lower pressures, but do not find its limits. The type of graphene coverage achieved is compared with the corresponding gaseous composition in equilibrium across the parameter space, to draw out the influence of various hydrocarbon species on deposition. Such analysis has proven useful for other, classic CVD systems such as SiC coatings. ${ }^{13}$ Our analysis allows the type of deposited graphene to be tailored through appropriate selection of CVD parameters, enabling reliable production of low-defect continuous monolayer films or isolated graphene regions either hexagonal or lobed in shape.

\section{RESULTS AND DISCUSSION}

Assumptions of the Model. A classical CVD approach is taken for this thermodynamic analysis, as described in the Methods section. It is important though, for the sake of discussion, to highlight the following assumptions made herein:

1. Kinetics is not considered in this model. Traditionally, CVD mechanisms are approached separately from equilibrium and reaction kinetic models, with one of these then considered the dominant driver. ${ }^{13}$ There has been a significant amount of detailed, good quality research into graphene kinetics, including nucleation and growth rates. This work adds the thermodynamic component so that the prime driver may be assessed. It should be noted that in the discussion of this Article, in order to fully understand the growth process, kinetic arguments from the literature are combined with our thermodynamic insights.

2. The model determines the gas phase equilibrium by minimizing the Gibbs free energy of the system for a given elemental composition, temperature, and pressure, using a well-established published algorithm and methodology ${ }^{24}$ developed and commercialized by the NPL, UK.

Parameter Space for Graphene Deposition. Graphene growth has been reported in hot wall CVD reactors over a range of temperatures, ${ }^{25-30}$ pressures, ${ }^{15,31,32}$ and gas feedstock compositions. ${ }^{27,33-37}$ We have classified the microstructure of each film reported using the following eight categories, which contain two groups showing either complete or partial graphene coverage. Complete coverage categories are (1) fewlayer graphene, (2) bilayer graphene, (3) decorated monolayer (multilayer islands covering $>5 \%$ of a continuous graphene monolayer), (4) monolayer, and (5) defective monolayer (the $\mathrm{sp}^{2}$ lattice is interspersed or overlaid with numerous $\mathrm{sp}^{3}$ regions). Partial coverage is classified as (6) interrupted monolayer (monolayer with breakages), (7) isolated islands (regions of monolayer or multilayer graphene, with diameters in the range of micrometers, which are unconnected on the copper surface), and (8) none (no detectable deposition). Where there is insufficient information to classify a reported graphene film, we have not used the data in the survey. For each set of growth conditions reported, $P_{\mathrm{A}}$ and $R_{\mathrm{CH}}$ were estimated by applying the ideal gas law to the flow ratio of the gas mixtures used. The published range of $R_{\mathrm{CH}}$ is between $2 \times 10^{-4}{ }^{33}$ and $0.67,^{30}$ and monolayer graphene has been produced for $R_{\mathrm{CH}}$ between $4 \times 10^{-4} \quad 33$ and $0.25^{38}$ (Supporting Information: Table S1). The parameter space for all reported $R_{\mathrm{CH}}$ is represented in Figure 1. Data points with $R_{\mathrm{CH}}<0.02$ almost always apply to atmospheric pressure CVD (APCVD) experiments, which use an inert gas to lower the partial pressure of the active species from the total reaction pressure, $P_{\mathrm{r}}$. The successful deposition of monolayer graphene with $R_{\mathrm{CH}}$ as low as $4 \times 10^{-4}{ }^{33}$ is discordant with recent work suggesting complete coverage is prevented by excessive hydrogen partial pressures, ${ }^{22}$ implying that the inert diluents used to lower $P_{\mathrm{A}}$ in APCVD may play a role in deposition.

From Figure 1 it is clear that, so far, very few experiments have used a $P_{\mathrm{A}}$ equal to atmospheric pressure. Where APCVD has been reported, a diluent gas is typically introduced to reduce $P_{\mathrm{A}}$ to between 10 and 100 mbar. It is unclear whether this lack of data is due to unreported null results for $P_{\mathrm{A}}=1013 \mathrm{mbar}$ or to lack of experiments in this region. For the low-pressure CVD (LPCVD) reactions, experiments have tended to 


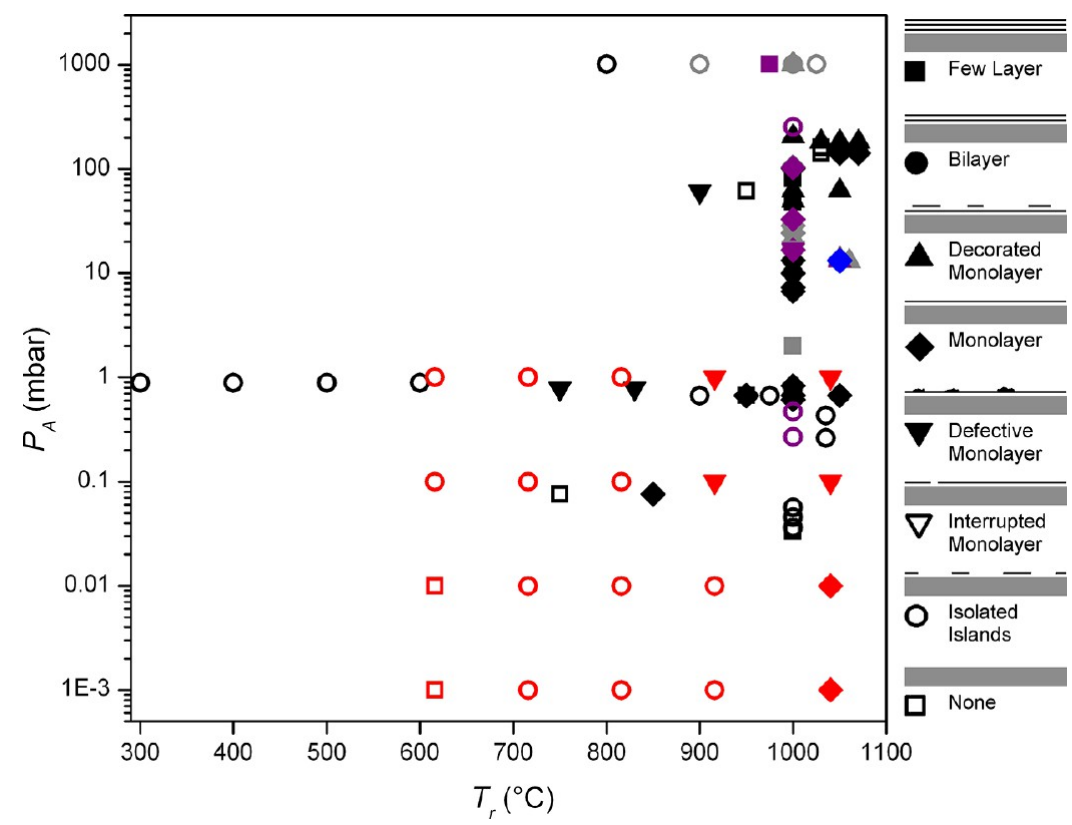

Figure 1. Coverage type for CVD of graphene in a hot walled furnace versus temperature and total active species partial pressure, $P_{\mathrm{A}}$, as reported in the literature., $15,25-30,32-36,38-51$ Note that these points span a range of different $R_{\mathrm{CH}}$ denoted by the color of the data points: black for $1>R_{\mathrm{CH}}>0.1$; gray for $0.1>R_{\mathrm{CH}}>0.01$; purple for $0.01>R_{\mathrm{CH}}>0.001$, blue for $0.001>R_{\mathrm{CH}}>$ 0.0001 . The red points correspond to data collected in this work, all of which have $R_{\mathrm{CH}}=0.2$. The inset is a schematic showing the coverage types defined in this study and the corresponding symbols assigned for scatter plots.

use a pressure close to that reported in 2009 by Li et al. $\left(P_{\mathrm{A}}=0.1\right.$ to 1 mbar). ${ }^{7}$ No deposition of continuous films has been reported for $R_{\mathrm{CH}}<0.1$ at these low $P_{\mathrm{A}}$. Deposition of monolayer graphene has not been reported to date for $P_{\mathrm{A}}<0.07$ mbar. ${ }^{29}$

Figure 1 shows that the data points from the literature are clustered around deposition temperatures of $1000{ }^{\circ} \mathrm{C}$, similar to the report of Li et al. ${ }^{7}$ These conditions usually lead to good quality monolayer graphene coverage. Reducing the temperature tends to form more defective or discontinuous films. To the best of our knowledge, the lowest temperature used for CVD of graphene on copper is $300^{\circ} \mathrm{C}$ using toluene as a feedstock, leading to the deposition of isolated graphene islands. ${ }^{30}$

Expanding the Parameter Space to Lower Pressures. In order to better understand the thermodynamics of growth, the parameter space explored for CVD of graphene needs to be extended to lower pressures. Hot wall CVD was conducted at temperatures of 716 , 816,916 , and $1040{ }^{\circ} \mathrm{C}$, and the deposition total pressure was held at order of magnitude intervals between 1 and 0.001 mbar for each temperature used. $R_{\mathrm{CH}}$ was set to 0.2 by using a methane:hydrogen feedstock of 2:1 by volume, in order to be comparable with the modal $R_{\mathrm{CH}}$ reported in the literature (Figure 1, Supporting Information: Table S1). No inert diluents were used; hence $P_{\mathrm{A}}=P_{\mathrm{r}}$. These new points are plotted in red in Figure 1 . The graphene films were analyzed to determine the appropriate film category without transferring the film from the copper substrate, to eliminate any artifacts introduced by the transfer process.
Ultrahigh-resolution scanning electron microscopy (SEM) images were collected using a Sirion FEGSEM with a through-lens secondary electron detector. The SEM images in Figure 2 show that total graphene coverage was attained at all pressures with a deposition temperature of $1040{ }^{\circ} \mathrm{C}$, with the highest quality films obtained at 0.001 mbar; this is the lowest controlled pressure reported for hot walled CVD of graphene to the authors' knowledge. Raman spectra obtained from the graphene on the copper substrate confirmed the monolayer nature of the graphene films (Supporting Information: Figure S1). Complete coverage was also achieved at $916^{\circ} \mathrm{C}$ when using the higher deposition pressures of 1 and 0.1 mbar (Figure 2 (c) and (g)). However, the films deposited at these pressures tended to have a greater incidence of defects and sooty deposits (Figure 2 (a)-(h)), which appear darker and have amorphous, pockmarked textures in the SEM images (Supporting Information: Figure S2). All other experiments with $T_{\mathrm{r}}>616^{\circ} \mathrm{C}$ resulted in deposition of isolated islands of graphene. At $T_{\mathrm{r}}<916^{\circ} \mathrm{C}$ the islands were frequently surrounded by a sooty region, which, at the higher deposition pressures of 0.1 and $1 \mathrm{mbar}$, often extended close to the center of the graphene islands. Black speckles (low secondary electron emitting regions) are often seen between the islands for such samples. Interestingly, the resonant graphene signal appears to dominate over the defect signal in the Raman spectra, until $T_{\mathrm{r}}<816^{\circ} \mathrm{C}$ (Supporting Information: Figure S1). The graphene island shape tends to be lobed at lower pressures, becoming equiangular as pressure is increased and temperature is reduced. 

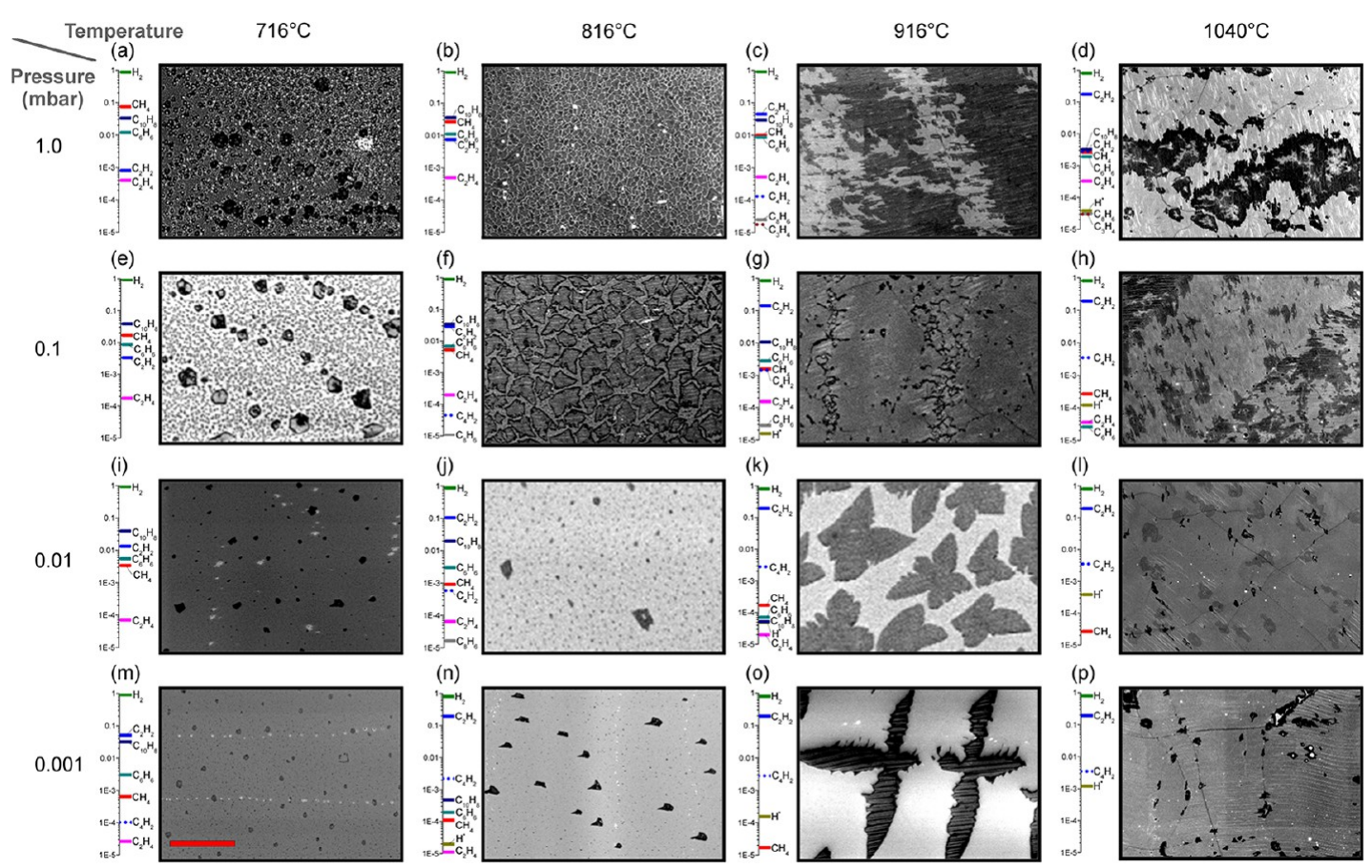

Figure 2. SEM images of graphene grown at various temperatures and pressures with $R_{\mathrm{CH}}=0.2$. Insets show the fractional equilibrium composition of the gaseous phase for the experimental conditions used for each sample. The gaseous phase compositions have been curtailed to species comprising at least $10^{-5}$ of the mixture for clarity. Scale bar: $10 \mu \mathrm{m}$.

Note that the defects and adlayers of soot were significantly more difficult to detect when using the standard secondary electron detector compared to the UHR in-lens detector of the SEM (Supporting Information: Figure S2). In the majority of literature it is unclear what type of detector was used in the SEM analysis and hence how clearly the sooty deposits would appear in the micrographs.

We have found that optical dark field microscopy (ODFM) provides a particularly clear, high-contrast image of graphene films grown on copper (Figure 3 ). Although ODFM is a straightforward imaging technique that is widely used to characterize mechanically exfoliated graphene, to the authors' knowledge there are no reports of ODFM imaging of CVD graphene films on the growth substrate. The graphene preserves the faceted structure adopted by the copper surface at the temperatures and pressures used for deposition. ${ }^{52,53}$ These rough areas diffract incident light, and so regions of the substrate with a graphene coating are clearly visible in the ODFM micrographs, for example in Figure $3(\mathrm{k}),(\mathrm{l}),(\mathrm{o})$, and (p). Graphene islands with diameters down to $0.7 \mu \mathrm{m}$ have been imaged using ODFM (Figure 3 (a) (b), (e), (f), (i)-(k), and (m)-(o)). Figure $3(k)$ and $(m)$ in particular show a striking resemblance to the structures visible in the SEM micrographs of the same samples (Figure $2(k)$ and $(m)$ ). Sooty deposits appear brighter than the underlying graphene when they have a filament morphology (Figure $3(\mathrm{~g})$ ) and obscure light from the dark field with more extensive coverage (Figure $3(c)$ and $(d)$ ). Optical dark field microscopy therefore provides a large-area, rapid, and nondestructive preliminary characterization technique, useful for indicating deposition type and identifying regions of interest for further analysis using more indicative but higher spatial resolution techniques such as Raman spectroscopy.

Thermodynamic Model and the Relevance of Gas Flow Conditions. Figures $1-3$ show that the deposition temperature and pressure influence the quality and coverage of the graphene film. The reactant gas composition also effects deposition, since $R_{\mathrm{CH}}<0.02$ for APCVD seems to consistently lead to complete surface coverage. The temperature, pressure, and elemental composition of a closed system define its thermodynamic state; hence we examined the equilibrium composition of the gas in a hot wall graphene CVD system.

Graphene film growth through CVD is, by definition, a nonequilibrium process. However, it is instructive to consider two limiting cases, ${ }^{13}$ schematically illustrated in Figure 4: (1) the equilibrium in the gas phase far from the copper substrate and (2) the equilibrium above a stable graphene film. Case 1 gives the equilibrium composition of the gas in the bulk of the reactor where no graphene can form. Case 2 represents the gas composition above a growing graphene film, where the velocity of gas molecules approaches zero as they stick to the substrate. The difference in composition between the two regimes drives carbon transport to the surface. The classical approach to mass transport in CVD systems assumes that the diffusion of species between cases 1 and 2 occurs across a stagnant 

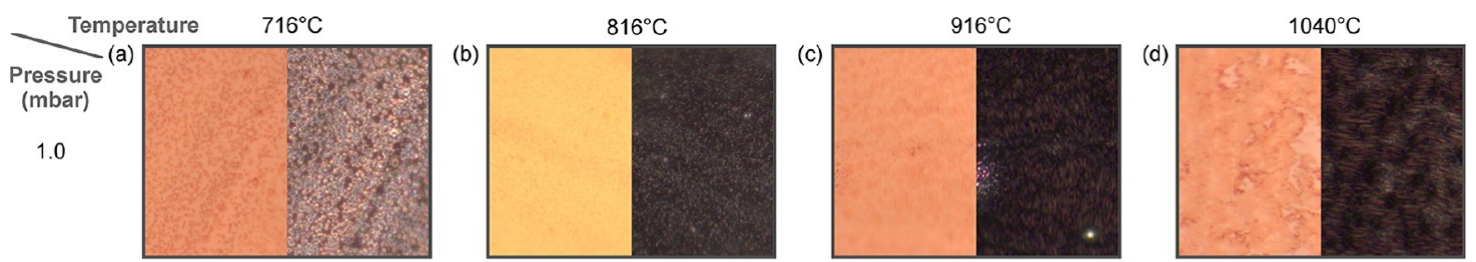

0.1
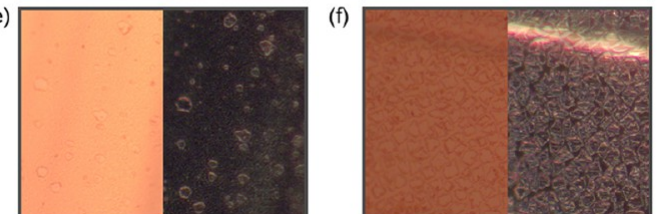

(g)
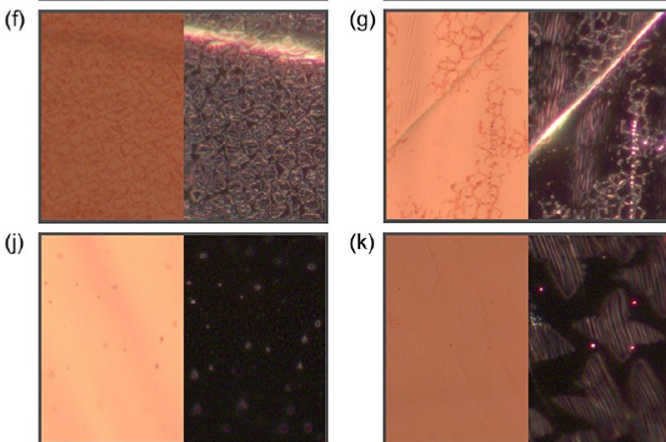

(i)
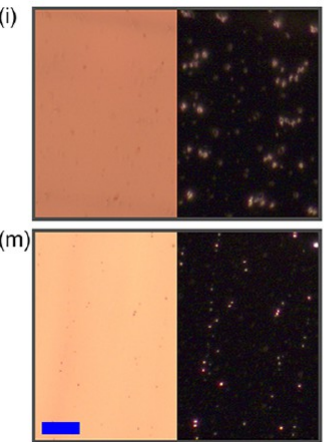

(n)

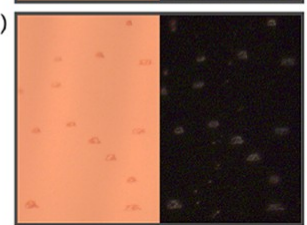

(k)

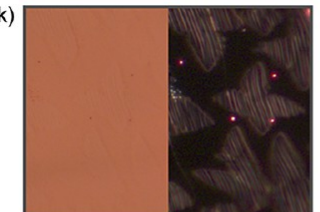

(o)

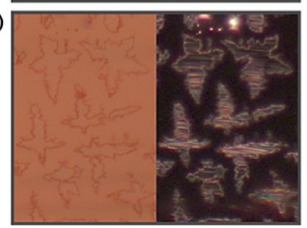

(h)

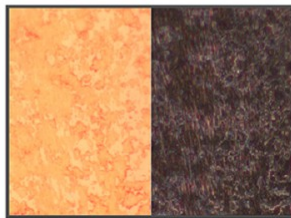

(I)

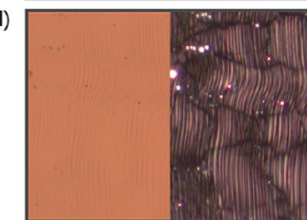

(p)

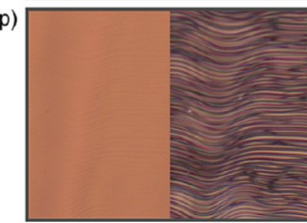

Figure 3. Composite optical microscope images (left image, bright field; right image, dark field) of CVD graphene films on copper substrates grown across a range of temperatures and pressures. Scale bar: $10 \mu \mathrm{m}$.

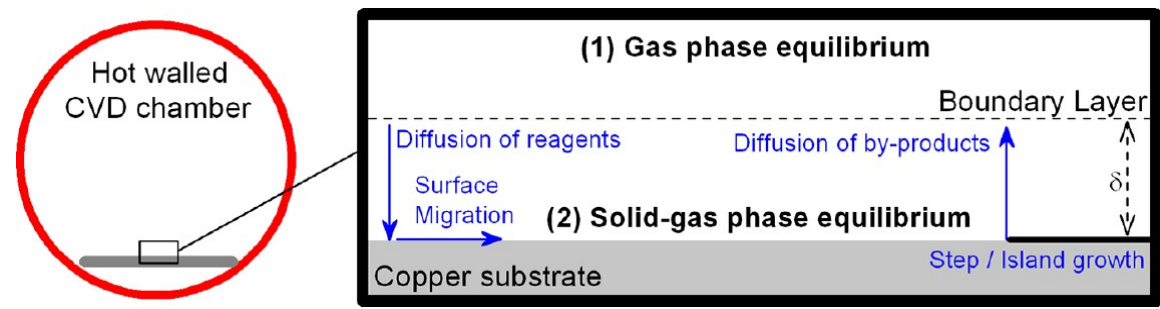

Figure 4. The classical, simplified view of CVD showing the two phase conditions that apply, not taking into account variations in boundary layer thickness or the rate of surface processes such as adsorption, decomposition, migration, incorporation into the growing lattice, and desorption of byproducts. ${ }^{13}$

boundary layer of the substrate in a laminar flow, schematically illustrated in Figure 4.

The boundary layer is defined for viscous flows as the layer of gas across which the flow velocity increases from zero at a flat surface to its maximum value in the main body of the flow. When this layer is thin, the parabolic concentration profile where cases 1 and 2 mix can be approximated as linear, and Fick's law is used to model diffusion across it between the two cases. The average thickness of the boundary layer over a substrate of length $L_{s}\left\langle\delta\left(L_{s}\right)\right\rangle$, is related to the Reynolds number according to eq 1 :

$$
\left\langle\delta\left(L_{s}\right)\right\rangle=\frac{10 L_{s}}{3\left(\operatorname{Re}\left(L_{s}\right)\right)^{1 / 2}}
$$

${ }^{14}$ where the Reynolds number, $\operatorname{Re}(L)$, is calculated using eq 2:

$$
\operatorname{Re}(L)=\frac{\rho v L}{\mu}
$$

with $\rho=$ gas density, $v=$ velocity, $L=L_{s}$, and $\mu=$ dynamic viscosity. From this it can be seen that, for an ideal gas,
$\left\langle\delta\left(L_{s}\right)\right\rangle$ varies with mass flow rate and composition but not with total gas pressure (see the Supporting Information for further details on the calculations).

In order to assess the validity and limitations of applying a gas composition model based upon these two bounding cases, it is useful to consider some of the properties of the gas flow through the reactor. For these calculations, we have assumed an ideal gas entirely comprising the main species for simplicity. Since the properties of the gas flow are closely related to the reactor dimensions, we conduct a complete analysis for our conditions and apply our geometry to approximate the flow conditions reported in the literature. More detail on the calculations can be found in the Supporting Information.

The model assumes that flow is viscous and laminar and can be represented as a continuum. When the total chamber pressure is $\geq 1 \mathrm{mbar}$ (as is the case in most of the prior published data presented in Figure 1), the gas conditions in a CVD reactor are in the viscous 
regime and thermodynamic equilibrium calculations are appropriate. However, reducing the system pressure to $P_{\mathrm{r}}=0.1$ mbar incurs a transition in the flow, so that molecular flow is established when $P_{\mathrm{r}} \leq 0.01 \mathrm{mbar}$. The continuum model breaks down for these low pressures as, inside the reactor, the molecules of the gas are widely separated and collide infrequently. The Reynolds numbers for graphene CVD are consistently below the critical value for the onset of turbulence (Supporting Information: Table S2), so laminar flow is always expected. However the constraints imposed by the reactor tube geometry prevent the formation of a stagnant boundary layer for all but the highest reported flows of $1500 \mathrm{sccm}$. Thus for the majority of the graphene CVD parameter space, cases 1 and 2 are bounding limits for the gas phase chemistry away from and close to the substrate, rather than absolute descriptions of the composition.

Undertaking deposition at atmospheric pressure leads to sufficiently long residence times for equilibrium to be established (see the Supporting Information for further details). For LPCVD, the time taken to achieve equilibrium may be lower than the residence time in the reactor. The methane feedstock is therefore expected to act as a carrier for gases formed under equilibrium conditions when the deposition is undertaken at low pressures. Although the equilibrium composition will only comprise a small fraction of the LPCVD environment, the amount of carbon contained therein is still several orders of magnitude greater than that required to coat the substrate with graphene (see the Supporting Information for further etails). The stable gases in equilibrium are therefore still significant, even assuming only a small fraction can adhere to the substrate and influence deposition.

Thermodynamic Analysis of the Equilibrium Gas Compositions. The gas phase equilibrium compositions for case 1, far from the substrate, are presented in Figure 5 across a range of $P_{\mathrm{A}}$ for $R_{\mathrm{CH}}=0.2$. At low temperatures, methane is the most abundant hydrocarbon for all pressures considered. Increasing the temperature to $1000{ }^{\circ} \mathrm{C}$ increases the concentration of large and unsaturated hydrocarbons in the gas mixture, so that $\mathrm{C}_{10} \mathrm{H}_{8}$ and $\mathrm{C}_{6} \mathrm{H}_{6}$ become available for deposition (Figure $5(\mathrm{a})-(\mathrm{d})$ ). Reducing $P_{\mathrm{A}}$ below 1 mbar reduces the concentration of the large hydrocarbons as the temperature is increased beyond $800{ }^{\circ} \mathrm{C}$, where acetylene becomes the most stable hydrocarbon at typical deposition temperatures (Figure 1, Figure 5 (e), (f)). The hydrogen radical markedly increases in abundance at the lower deposition pressures investigated.

The equilibrium compositions of the gas mixture for case 2, where the graphene film can form from the hydrocarbon atmosphere, are presented in Figure 6 $\left(R_{\mathrm{CH}}=0.2\right)$. To make these calculations, we assume that, in the absence of other data, the free energy of graphene can be approximated by that of graphite (see Methods section for further details). Methane is the most abundant form of carbon in case 2 at low temperatures, as was found for case 1 . With a small increase in temperature, deposition of graphene becomes thermodynamically favorable, since in equilibrium the solid phase consumes the majority of carbon in the system. The effect of reducing pressure has little influence on the species stable in case 2, other than for the highest pressures used for graphene CVD at $R_{\mathrm{CH}}=0.2$, where several hydrocarbons can be present in low quantities (Figure $6(\mathrm{a})$ ). As pressure is reduced, methane stability drops more rapidly with increasing temperature and the likelihood of forming the hydrogen radical improves. The pressure sets the minimum thermodynamically feasible temperature for deposition, indicated by a dashed line in Figure 6. At $100 \mathrm{mbar}$ the solid phase cannot be deposited below $350{ }^{\circ} \mathrm{C}$ (Figure $6(\mathrm{a})$ ). Reducing $P_{\mathrm{A}}$ to $0.001 \mathrm{mbar}$ decreases the minimum theoretically possible deposition temperature to $90^{\circ} \mathrm{C}$ (Figure $6(\mathrm{f})$ ).

Driving Force for Deposition. The carbon solubility of a gaseous system of hydrocarbons, $C_{\text {sol, }}$ is defined as the number of moles of atomic carbon contained in gaseous species divided by the number of moles of atomic hydrogen. ${ }^{54}$ Away from the substrate, $C_{\text {sol_1 } 1}=R_{\mathrm{CH}}$, since all of the carbon is in the gas phase. The carbon solubility for case $2, C_{\text {sol_2 }}$, was calculated using the output from the thermodynamic model. The difference in the carbon solubility between cases 1 and $2, \Delta C_{\text {sol }}=$ $R_{\mathrm{CH}}-C_{\text {sol_2 }}$, represents the driving force for carbon transport from the bulk of the gas to the growing film at the substrate surface. For example, when the conditions give a $\Delta C_{\mathrm{sol}}$ at its maximum value of $\Delta C_{\mathrm{sol}}=R_{\mathrm{CH}}$, $C_{\text {sol_2 }}$ must be zero. Under these conditions no carbon is present in the equilibrium gas phase near the growing film, resulting in a high diffusion gradient for carboncontaining molecules traveling from case 1 into case 2 . Conversely, when $\Delta C_{\text {sol }}=0$, no carbon is stable in the solid phase and, from a thermodynamic perspective, deposition is impossible. $\Delta C_{\text {sol }}$ is plotted in Figure 7 across the parameter space used for graphene CVD for all conditions with $R_{\mathrm{CH}}=0.2$.

Figure 7 shows that $\Delta C_{\text {sol }}$ varies only slightly over the temperature and pressure range used for graphene deposition, resulting in a carbon-rich environment near the substrate surface. This opens a wide range of conditions across which deposition is thermodynamically possible and kinetically feasible, as demonstrated in both the literature and our work. All of the reported deposition has $\Delta C_{\text {sol }}>0.19$, which approaches the maximum possible value of $\Delta C_{\mathrm{sol}}=R_{\mathrm{CH}}$, so the driving force for deposition is extremely high. When the data are replotted for other $R_{\mathrm{CH}}$, the general trend remains similar. A significant consequence of these calculations is that given the relatively constant carbon solubility difference, the lack of formation of a 

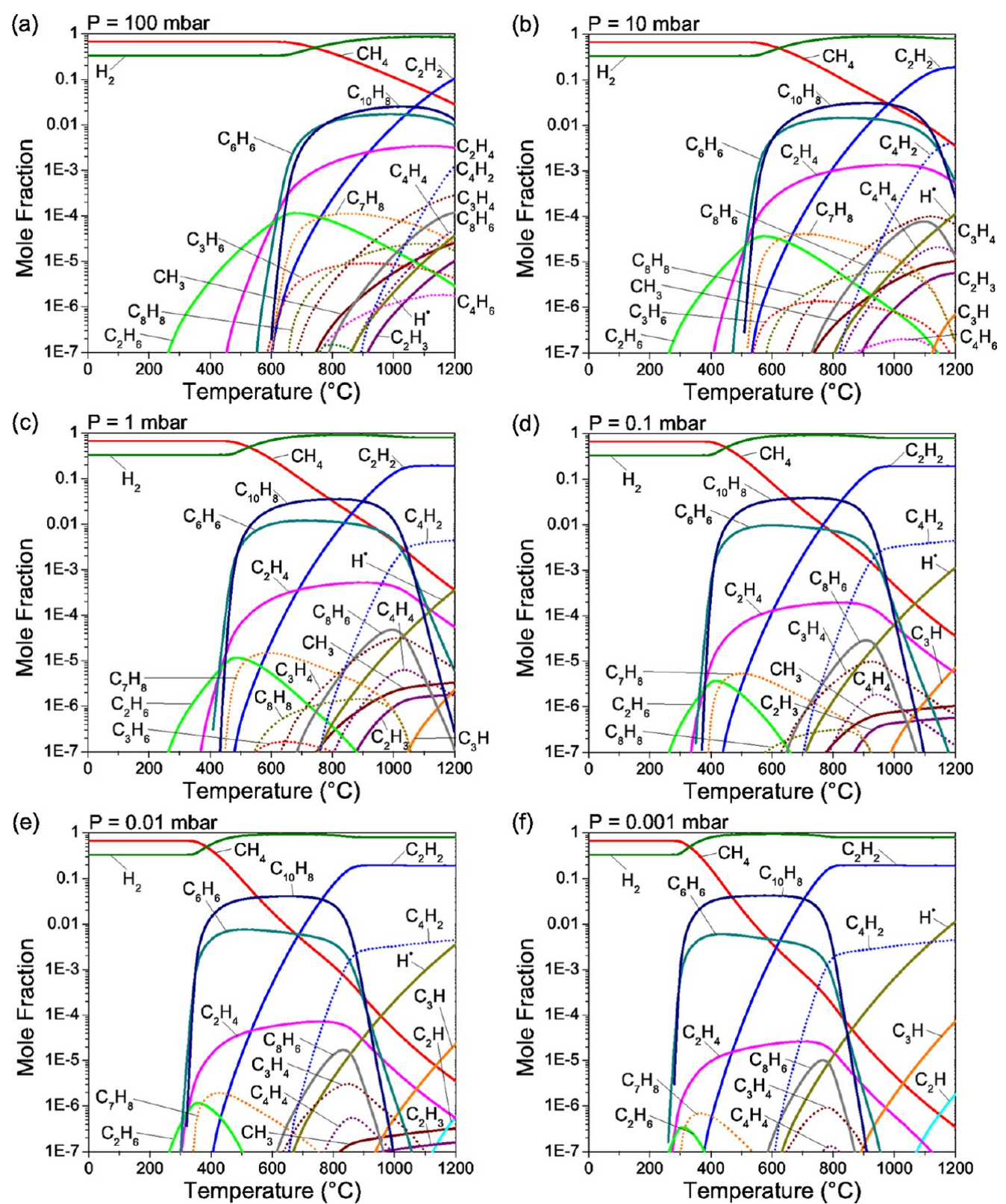

Figure 5. Equilibrium gas phase composition away from the copper substrate (case 1) for $R_{\mathrm{CH}}=0.2$ and active species pressures of (a) 100 mbar, (b) $10 \mathrm{mbar}$, (c) $1 \mathrm{mbar}$, (d) $0.1 \mathrm{mbar}$, (e) $0.01 \mathrm{mbar}$, and (f) $0.001 \mathrm{mbar}$.

stagnant boundary layer for the majority of CVD conditions, and the fact that gaseous diffusion shows a small dependence on temperature, mass transport is unlikely to be the reason for the variations in the deposited graphene films. ${ }^{13,15}$

Influence of Gas Phase Chemistry on Graphene Growth. As discussed in the model validity section, cases 1 and 2 represent the two bounds of the thermodynamic equilibrium composition. The composition of case 2 is reasonably constant with pressure and temperature (Figure 6), with the only significant changes for $T_{r} \geq 1000^{\circ} \mathrm{C}$ being an increase in monatomic hydrogen and a decrease in the onset temperature for acetylene stabilization as the system pressure is lowered. The proposed catalytic effect of the copper substrate will accelerate the development of case 2 equilibrium, which is enhanced further when monatomic hydrogen can form. ${ }^{22,36}$

For $R_{\mathrm{CH}}=0.2$, monolayer coverage is reported when $T_{\mathrm{r}} \geq 1000{ }^{\circ} \mathrm{C}$ and $P_{\mathrm{A}} \geq 10 \mathrm{mbar}$, where no hydrocarbons other than methane and acetylene are stable near the graphene film (Figure 6 (a) and (b)). The graphene quality improves as $P_{\mathrm{A}}$ is reduced, which causes the stability of acetylene relative to methane to rise (Figure $6(\mathrm{a})-(\mathrm{d})$ ). The SEM and Raman spectroscopy results show that decreasing $P_{\mathrm{A}}$ further to 0.001 mbar results in the best quality films (Figure 2 and 3 (p), Supporting Information: Figure S1), although this is where the limitations of the model are reached since for such experiments molecular flow applies. It may be reasonable to assume that the trend continues so that 

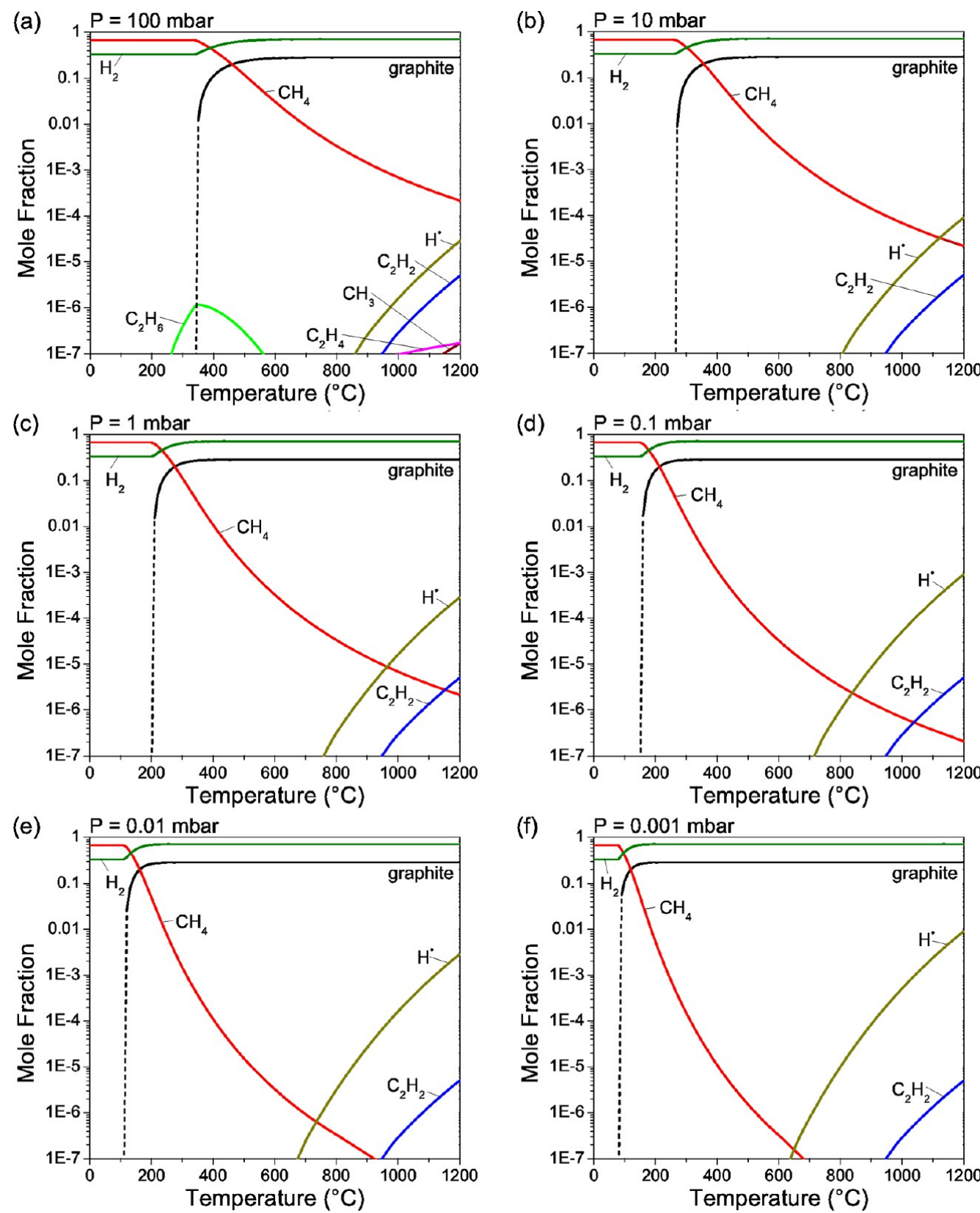

Figure 6. Gas phase composition in equilibrium with solid graphite (case 2) for $R_{\mathrm{CH}}=0.2$ and active species pressures of (a) 100 mbar, (b) 10 mbar, (c) 1 mbar, (d) 0.1 mbar, (e) $0.01 \mathrm{mbar}$, and (f) $0.001 \mathrm{mbar}$.

the partial pressure of acetylene exceeds that of methane for the best quality films (Figure $6(\mathrm{f}), T_{\mathrm{r}}=1040{ }^{\circ} \mathrm{C}$ ); however more involved computational techniques such as Monte Carlo simulations would be required to determine the gas phase composition under these conditions.

The importance of acetylene for graphene deposition can be understood by considering the reaction kinetics; as an unsaturated hydrocarbon, acetylene has a higher probability of adsorbing onto the substrate surface than methane, ${ }^{55}$ and theoretical studies have demonstrated that a carbon dimer is more energetically favorable and more mobile on a copper surface than a carbon monomer. ${ }^{56-58}$ Furthermore, an acetylene-rich environment is believed to be important for the growth of single-walled carbon nanotubes, ${ }^{59}$ a carbon allotrope closely related in structure to graphene. The acetylene may then go on to form the carbon clusters recently proposed as precursors for graphene growth. ${ }^{22,60}$

Conversely, the methane concentration in case 2 seems to have little influence on the deposited graphene. Its concentration changes over several orders of magnitude for total film coverage. Furthermore in an experimental CVD reactor, given the residence times and gas reaction kinetics, ${ }^{61}$ it is likely that methane constitutes $>99 \%$ of the hydrocarbon species. A wide range of coverage types is obtained for these experiments, suggesting that methane 


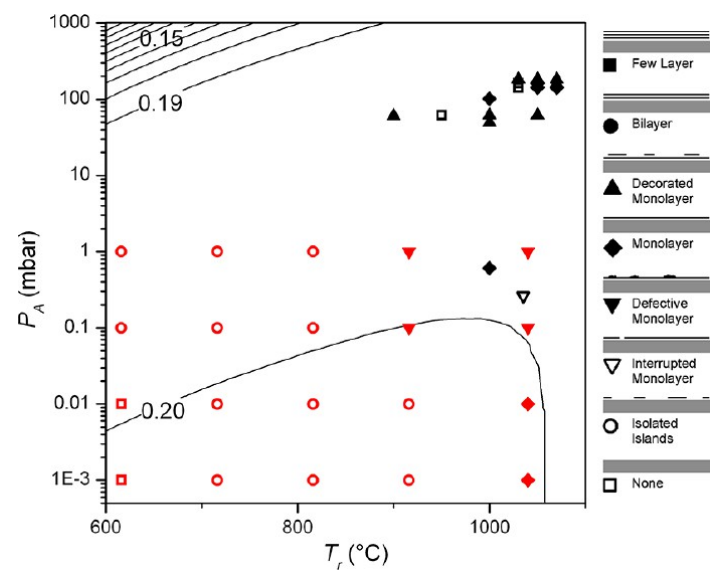

Figure 7. Contour map showing the difference in carbon solubility between cases 1 and $2, \Delta C_{\mathrm{sol}}$, with $R_{\mathrm{CH}}=0.2$. The overlying scatter plot shows experimental data points from Figure 1, whose $R_{\mathrm{CH}}$ lies between 0.18 and $0.22,15,27,34,40,41,43,45,48$ and from this work, where $R_{\mathrm{CH}}=0.2$ (red data points). The schematic showing the type of coverage represented by each data point is reproduced from Figure 1.

concentration is unlikely to be a controlling factor in the transport of carbon to the growing graphene films.

Case 1 shows more variation than case 2, with Figure 8 (a) giving the most abundant hydrocarbon by mole fraction, assuming equilibrium. It is apparent that continuous graphene growth is achieved under conditions where small molecules are most prevalent at the case 1 limits (Figures 2 and 3, (c), (d), (g)-(i), and (p)). The percentage of hydrocarbons stable as acetylene, methane, and benzene plus naphthalene are plotted in Figure 8 (b) - (d) to provide a more detailed understanding of the influence of different species formed in case 1 on graphene deposition. Figure 8 (b) shows that continuous coverage films (solid symbols) always have an equilibrium acetylene concentration $>1.2 \%$ of the hydrocarbons, while good quality monolayer films (solid diamonds) are reported only when $>5.6 \%$ of the hydrocarbons are stable as acetylene. The methane equilibrium concentration, however, varies over several orders of magnitude for continuous deposition, shown in Figure 8 (c). This behavior is similar to that observed at the case 2 limit.

In the region of Figure 8 (a) where naphthalene is the most stable hydrocarbon species, qualitative SEM analysis found that sooty deposits were common (Figure 2). Large, unsaturated hydrocarbons adhere well to surfaces ${ }^{55}$ and are associated with the deposition of pyrolytic carbon (soot). ${ }^{62}$ The percentage of hydrocarbons stable as large molecules (naphthalene and benzene) is presented in Figure $8(\mathrm{~d})$. The overlying scatter plot shows that, as expected, deposition of discontinuous and defective films becomes more common with an increased stability of large hydrocarbons. Sooty deposits were observed for all of the films deposited in this work using conditions where at least $1 \%$ of the hydrocarbons are stable as large molecules (red inverted triangles and red barred circles). When these molecules are at their most stable, no deposition is observed $(87 \%$ of the hydrocarbon environment at $616^{\circ} \mathrm{C}$ and $0.001 \mathrm{mbar}$ ); instead only black speckling was seen using SEM. This speckling disappears for $P_{\mathrm{A}}=0.001 \mathrm{mbar}$ at a deposition temperature of $916^{\circ} \mathrm{C}$ (Figure 2 (o)), where the stability of benzene and napthalene is negligible and where development of these molecules is further hindered by the inhibition of equilibrium formation in case 1 . Note that at the highest reported $P_{\mathrm{A}}$ the hydrocarbon feedstock is often heavily diluted in hydrogen (Figure 1, Table S1), which suppresses formation of heavier hydrocarbons in case 1 so that deposition of clean films is still feasible. Conditions that result in low stability of large hydrocarbons concurrently promote the formation of the hydrogen radical, $\mathrm{H}^{*}$, in both case 1 and case 2 (Figures 5 and 6 ), further suppressing the agglomeration of sooty deposits by enhancing hydrocarbon decomposition. ${ }^{36,63}$

Effect of Inert Gas Diluents and $\boldsymbol{R}_{\mathrm{CH}}$ on Graphene Deposition. Using inert gas diluents in APCVD extends the residence time by providing a high $P_{r}$, allowing equilibrium to develop, while also providing the low $P_{\mathrm{A}}$ required to stabilize acetylene (Supporting Information: Figure S3 (a) and (b)). Under these conditions, continuous deposition is frequently reported (Figure 1, Supporting Information: Table S1), in agreement with the correlation between increased acetylene stability and continuous film deposition established above. The increase in $P_{r}$ will also decrease the vaporization of copper from the growth substrate.

In APCVD experiments, film quality tends to improve for hydrocarbon flows more heavily diluted in hydrogen, indicating that deposition can be controlled by tuning $R_{\mathrm{CH}}$. For example, Wu et al. observed an improvement in the coverage and an increase in the number of layers of graphene deposited by raising $R_{\mathrm{CH}}$ from 0.0002 , where the graphene film was interrupted by areas of bare copper, to $0.0004<R_{\mathrm{CH}}<0.0011$, where monolayer graphene was obtained, to $R_{\mathrm{CH}}=$ 0.02 , which led to deposition of multilayer islands on the film. ${ }^{33}$ The equilibria for case 1 show that at the lowest $R_{\mathrm{CH}}$ used by Wu et al. the formation of large hydrocarbons is completely suppressed, and the partial pressure of acetylene is comparable to that of monatomic hydrogen at the $T_{\mathrm{r}}$ of $1050{ }^{\circ} \mathrm{C}$ (Supporting Information: Figure S3 (d)). The resulting interrupted film is consistent with the etching mechanism proposed for $\mathrm{H}^{\bullet} .^{22,36}$ Raising $R_{\mathrm{CH}}$ by 1 order of magnitude provides the desired gas phase chemistry by pushing the concentration of $\mathrm{H}^{\bullet}$ below that of acetylene while still inhibiting large hydrocarbon formation (Supporting Information: Figure S3 (c)), and good quality films are deposited. At the highest carbon concentration used by Wu et al. of $R_{\mathrm{CH}}=0.02$, the concentration of large hydrocarbons becomes significant and $\mathrm{H}^{\bullet}$ formation is notably diminished (Supporting Information: Figure S3 
(a)
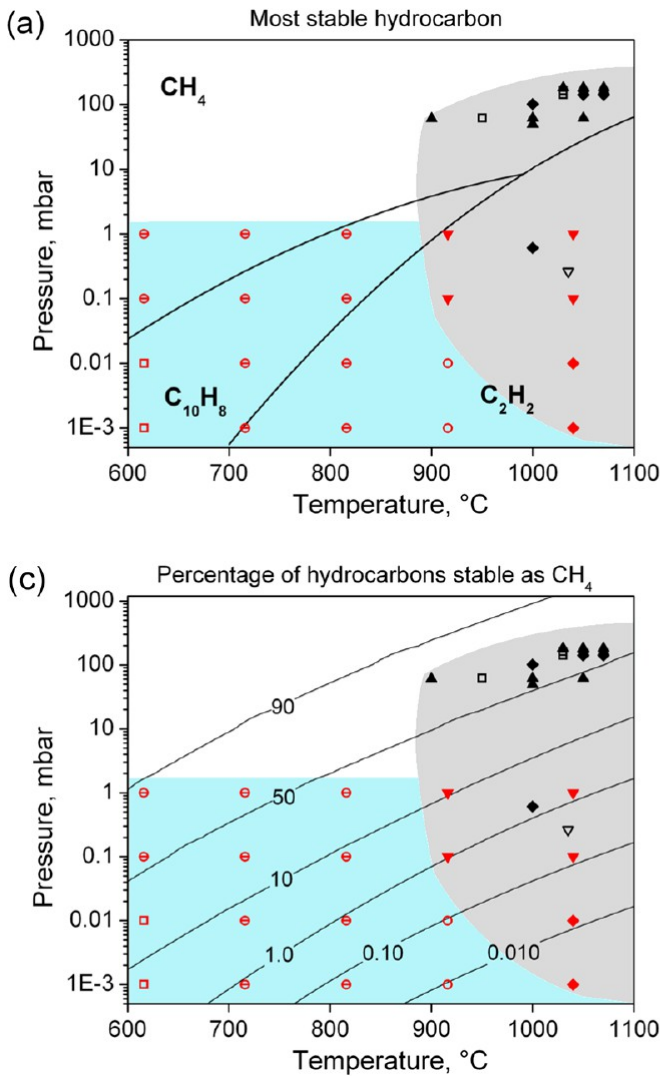

(b)

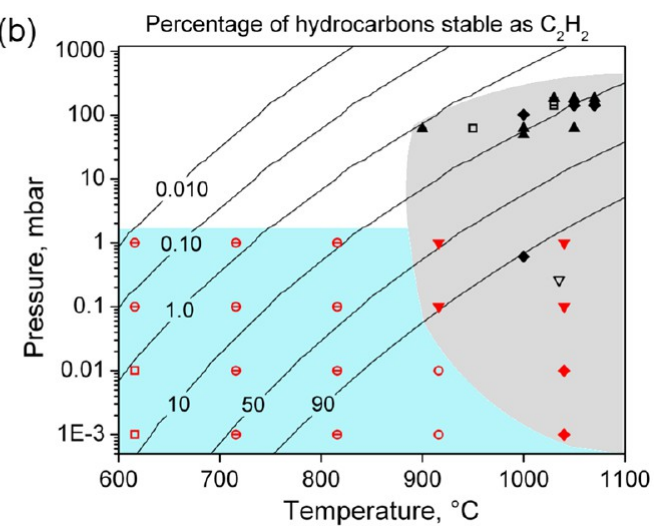

(d) Percentage of hydrocarbons stable as $\mathrm{C}_{6} \mathrm{H}_{6}+\mathrm{C}_{10} \mathrm{H}_{8}$

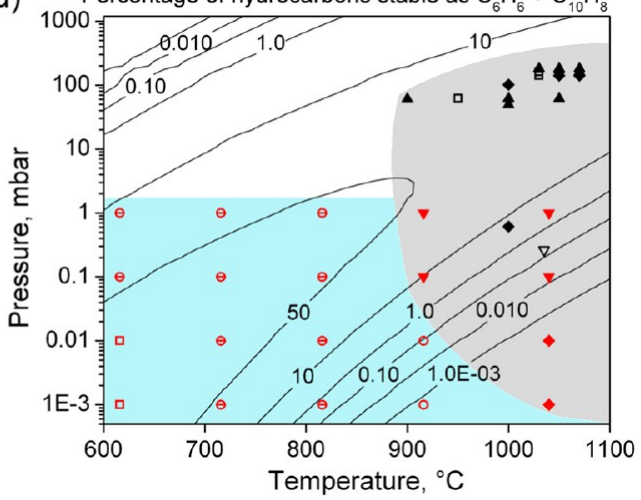

Figure 8. (a) The most stable hydrocarbons, determined by the species with the highest mole fraction in equilibrium, in case 1 for graphene CVD. Contour plots showing the percentage of hydrocarbons far from the copper substrate that are stable in case 1 as (b) acetylene, (c) methane, and (d) benzene plus naphthalene with $R_{\mathrm{CH}}=0.2$. The overlying scatter plot shows experimental data points from Figure 1 , whose $R_{\mathrm{CH}}$ lies between 0.18 and $\mathbf{0 . 2 2}, 15,27,34,40,41,43,45,48$ and from this work, where $R_{\mathrm{CH}}=0.2$ (red data points). Barred circles indicate isolated islands with a sooty perimeter. The gray shaded area indicates where continuous coverage has been obtained, and the blue area where graphene islands are deposited.

(b)), so etching of the multiple graphene layers by the gas phase is less likely.

Effect of Equilibrium Gas Composition on Island Shape. Where graphene islands form, their shape can broadly be divided between two categories: those whose vertices coincide with those of the graphene atomic lattice $\left(60^{\circ}\right.$ and $120^{\circ}$, resulting in hexagons and squat star shapes, Figure 2 (a), (e), (i), and (m)) and lobed islands with more complex edge structures (Figure $2(\mathrm{k})$ and (o)). This shape variation is frequently attributed to the concentration of hydrogen in the feedstock supply; when the concentration is high, the etchant effect of hydrogen on carbon is thought to lead to hexagonal island growth. ${ }^{36,37,64}$ Increased sublimation of the copper substrate at lower system pressures ${ }^{25}$ and the underlying orientation of the copper substrate $e^{65,66}$ have also been suggested as causes of the variation in island shape. Figure 9 provides an intriguing additional explanation. The distribution of hexagonal and lobed graphene islands is mixed across the range of partial pressures at which hydrogen is introduced to the furnace ( $x$ axis, Figure 9 (a) and (b)), so this is unlikely to be responsible for the observed variation in island shape. In this work, both forms of island are obtained for the same $P_{\mathrm{r}}$ (for example Figures $2(\mathrm{~m}$ ) and (o)), suggesting that copper evaporation does not control the graphene edge structure. Backscatter electron imaging and electron backscatter diffraction revealed that although the graphene islands produced in this work and in the literature can be distorted and stretched by different crystallographic textures in the underlying copper, their general form (lobed or hexagonal) was maintained. However, the island shape distribution correlates with the stability of $\mathrm{H}^{\bullet}$ at the substrate surface ( $y$ axes, Figure 9 (a) and (b)). Hexagonal islands are deposited when $<4 \times 10^{-4} \%$ of the CVD atmosphere is stable as $\mathrm{H}^{\bullet}$ in case 1 and $<3 \times$ $10^{-4} \%$ in case 2 , and at higher $\mathrm{H}^{\circ}$ partial pressures, lobed islands are usually observed. We propose that the stability of the hydrogen radical, rather than the ratio of hydrogen to methane introduced to the reaction chamber, contributes to the variation in island shape.

We expect the hydrogen radical to create dangling bonds at the edge of the growing graphene film and on the copper surface, increasing the number of sites available for carbon attachment in line with the mechanism for amorphous carbon formation during 
(a)

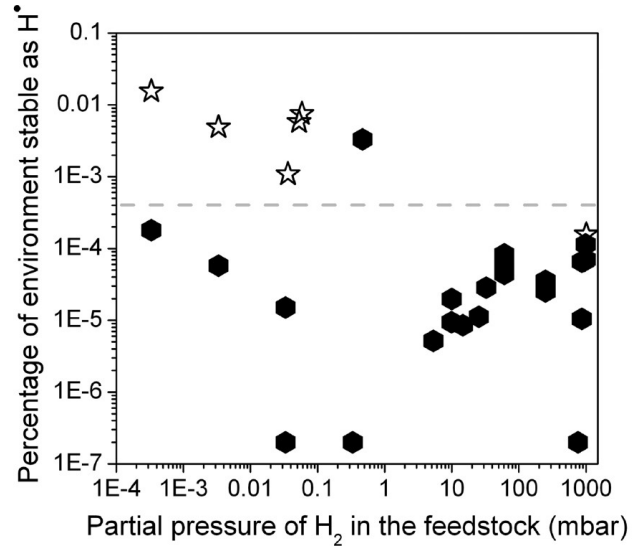

(b)

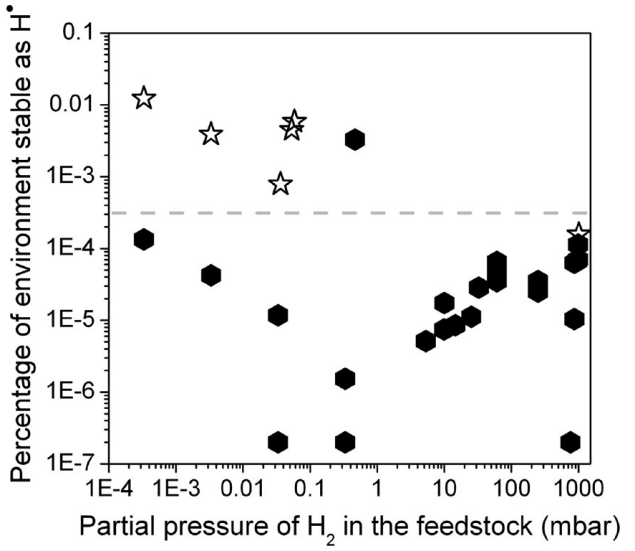

Figure 9. Percentage of the CVD environment stable as $\mathrm{H}^{\bullet}$ for (a) case 1 and (b) case 2 versus the partial pressure of hydrogen introduced to the furnace for this work and in the literature, where the shape is easy to distinguish. $25,27,36,42,48-50,65,69-71$ The $R_{\mathrm{CH}}$ of data points in this figure lies between $0.0001^{70}$ and $0.24 .{ }^{49}$ The shape of the data points corresponds to the shape of deposited graphene islands, with stars for lobed islands and hexagons for islands whose vertices follow that of the graphene lattice. Data points where the equilibrium pressure of $\mathrm{H}^{*}$ is zero have been given a false value of $2 \times 10^{-7} \%$ to allow their inclusion in this figure. The gray dashed line indicates the apparent maximum limit on the equilibrium pressure of $\mathrm{H}^{\circ}$ for the consistent production of hexagonal islands.

plasma deposition ${ }^{67}$ (note that $\mathrm{H}^{\bullet}$ is also known to play a crucial role in activating sites for adatom bonding in CVD of diamond $\left.{ }^{63,68}\right)$. Zhang et al. have demonstrated that an exposed copper surface is required for etching of graphene by hydrogen. ${ }^{37}$ From examination of the reaction kinetics Vlassiouk et al. conclude that the copper surface catalyzes the breakdown of $\mathrm{H}_{2}$ into $2 \mathrm{H}^{\circ}$, which then both promotes the decomposition of methane and etches the graphene. ${ }^{36}$ Our thermodynamic analysis supports and builds on this work. When the stability of $\mathrm{H}^{\bullet}$ is high, it can be formed more rapidly by the copper catalyst, providing a high concentration of $\mathrm{H}^{\bullet}$ near the growing graphene edge. We postulate that a high concentration of $\mathrm{H}^{*}$ opens multiple sites for carbon attachment, and lobed islands can form, growing along step edges in the copper or developing highly complex edge structures at the lowest $P_{\mathrm{A}}$ where the stability of $\mathrm{H}^{\bullet}$ is highest (Figure $\left.2(0)\right) .{ }^{31,69}$ When the stability of $\mathrm{H}^{\bullet}$ is low, the energy barrier to carbon attachment governs growth, so that carbon absorbs on armchair edges rapidly ${ }^{16,21}$ and hexagonal islands with zigzag edges are deposited. ${ }^{70}$

\section{CONCLUSIONS}

The CVD growth of graphene was analyzed in terms of thermodynamic equilibrium by applying classic CVD theory where two limiting cases for equilibria are considered in the system: case 1, the equilibrium in the gas phase, and case 2 , the equilibrium between the deposited material on the substrate and the gas in its immediate vicinity. Case 2 explained why the literature parameter space spans a wide range of temperatures and pressures, while both cases explained the variation in the deposited graphene under different experimental conditions and the consistent success of the method for reaction temperatures of $1000^{\circ} \mathrm{C}$ and above.
The solid phase consumes the vast majority of systemic carbon in case 2 over a large range of temperatures and pressures, opening a wide parameter space across which CVD of graphene can be achieved. However, the parameters chosen for deposition strongly influence the equilibrium chemical composition, in turn affecting the quality and coverage of the deposited graphene. High-quality films are consistently deposited where there is a significant pressure of acetylene and when the formation of the more complex gas phase chemistry of case 1 is hindered by the use of low-pressure systems. We have also noted a tendency to form soot when large hydrocarbons are stable. The deposition conditions should therefore be selected to avoid having large hydrocarbons dominant, by the use of high temperatures and low $R_{\mathrm{CH}}$ or low pressures. Our work determines which molecules are likely to be incident on the substrate, so can inform kinetic studies concerning the reaction pathway for graphene formation.

Our equilibrium calculations suggest that the presence of $\mathrm{H}^{\boldsymbol{*}}$ influences the CVD of graphene, as has been shown previously for the CVD of other carbon allotropes. The transition in island shape correlates with a critical concentration of $\mathrm{H}^{\circ}$, with hexagonal islands forming at low $\mathrm{H}^{\bullet}$ stabilities and lobed islands forming when the stability of $\mathrm{H}^{\bullet}$ is higher. While the presence of $\mathrm{H}^{\bullet}$ is useful to provide clean deposition and suppress the formation of multilayer films, excessive amounts are expected to lead to graphene etching, as discussed in other studies.

The assessment of the validity of the model to our experiments showed that only a small proportion of the environment can achieve equilibrium under the conditions used herein. While this is not problematic when coating small substrates, a better conversion rate would enable more precise control of deposition when scaling to the larger area graphene production desired 
for commercial applications. This can be achieved by using inert gas diluents or large-bore reactors $(d>$ $10 \mathrm{~cm}$ ) to increase residence times, which would also promote the formation and control of a stagnant boundary layer, allowing the delivery of carbon to the substrate to be more carefully regulated. We anticipate that our establishment of the parameter space in which good quality graphene can be grown will aid interpretation of results and be a useful tool for the design of new experiments.

\section{METHODS}

Sample Preparation and Characterization. Graphene samples were prepared by CVD in a hot walled tube furnace (Lenton Eurotherm) using $25 \mu \mathrm{m}$ thick copper foils (99.8\% Cu, Alfa Aesar $\# 13382$ ) as substrates and a quartz tube 1 in. in diameter for the CVD chamber. Foils were evenly positioned in the isothermal zone of the furnace, where the temperature varies by less than $1 \%$. Chamber pressure was reduced using a rotary vane pump (Edwards RV12), in conjunction with a turbomolecular pump (Leybold Oerlikon Turbovac50) for the lower pressures used, and monitored using a capacitance manometer gauge (Leybold Oerlikon CTR100). Deposition pressures between 1 and 0.001 mbar were controlled by pump selection and by setting the flow of hydrogen and methane through mass flow controllers (MKS Instruments). The copper foil was heated at $40{ }^{\circ} \mathrm{C} / \mathrm{min}$ to 1040 ${ }^{\circ} \mathrm{C}$ under a hydrogen atmosphere, and following a $30 \mathrm{~min}$ anneal the furnace was set to the desired deposition temperature. Once the chamber temperature had stabilized, the methane feedstock was introduced. After 30 min of growth, the methane flow was terminated and the furnace allowed to cool to room temperature before switching from hydrogen flow to nitrogen. The furnace thermostat was calibrated for the surface temperature of the copper foil by spot-welding a Type $\mathrm{K}$ thermocouple to the surface of the foil and heating in a lowpressure hydrogen atmosphere in a dummy run. All SEM micrographs were collected with the graphene in situ on the copper growth substrate at an accelerating voltage of $5 \mathrm{kV}$.

Thermodynamic Analyses. The composition for both case 1 and 2 can be calculated by iteratively minimizing the summed Gibbs free energy of formation of substances therein, scaled by their proportions, and by assuming equilibrium under the constraints of constant pressure, temperature, and $R_{\mathrm{CH}}$. In this case, the equilibria were computed using MTData, ${ }^{24}$ which is a commercial thermodynamic software package from the National Physical Laboratories. The equilibrium compositions are independent of the choice of feedstock, other than their influence on $R_{\mathrm{CH}}$. MTData's database contains data for $185 C_{1}$ to $C_{12}$ hydrocarbon and carbon species, all of which were permitted in calculations.

CVD of graphene on copper is a young field, and as such, a complete set of thermodynamic parameters for graphene on copper has not yet been produced. An alternative solid carbon phase must therefore be used for the equilibrium calculations. Pop et al. predict that the specific heat capacity of graphene approaches that of graphite as the temperature exceeds $100 \mathrm{~K}^{72} \mathrm{Gibbs}$ free energy is determined by integrating specific heat capacity with respect to temperature and adding to functions involving entropy and enthalpy. Therefore, unless the enthalpy and entropy contribution to the Gibbs free energy of graphene on copper differs significantly from that of graphite, the Gibbs free energy of the two materials will be very similar.

Conflict of Interest: The authors declare no competing financial interest.

Acknowledgment. This research was funded by the EPSRC. A.L. would like to acknowledge the NOWNano Doctoral Training Centre for funding and A. Wallwork for assistance with the design and optimiztaion of the CVD reactor.

Supporting Information Available: Table detailing the conditions for graphene CVD reported in the literature, characterization of the graphene samples on their copper substrates: Raman spectra; the appearance of sooty deposits in SEM micrographs; a normal secondary electron detector versus a through-lens detector for SEM, detailed information regarding the flow parameters and their calculation, and equilibrium compositions for carbon:hydrogen ratios other than 0.2 . This material is available free of charge via the Internet at http://pubs.acs.org.

\section{REFERENCES AND NOTES}

1. Novoselov, K. S.; Geim, A. K.; Morozov, S. V; Jiang, D.; Zhang, Y.; Dubonos, S. V; Grigorieva, I. V; Firsov, A. A. Electric Field Effect in Atomically Thin Carbon Films. Science 2004, 306, 666-669.

2. Soldano, C.; Mahmood, A.; Dujardin, E. Production, Properties and Potential of Graphene. Carbon 2010, 48, 21272150.

3. Guo, S.; Dong, S. Graphene Nanosheet: Synthesis, Molecular Engineering, Thin Film, Hybrids, and Energy and Analytical Applications. Chem. Soc. Rev. 2011, 40, 2644-2672.

4. Berger, C.; Song, Z.; Li, X.; Wu, X.; Brown, N.; Naud, C.; Mayou, D.; Li, T.; Hass, J.; Marchenkov, A. N.; et al. Electronic Confinement and Coherence in Patterned Epitaxial Graphene. Science 2006, 312, 1191-1196.

5. Obraztsov, A. N.; Obraztsova, E. A.; Tyurnina, A. V.; Zolotukhin, A. A. Chemical Vapor Deposition of Thin Graphite Films of Nanometer Thickness. Carbon 2007, 45, 2017-2021.

6. Yu, Q.; Lian, J.; Siriponglert, S.; Li, H.; Chen, Y. P.; Pei, S.-S. Graphene Segregated on Ni Surfaces and Transferred to Insulators. Appl. Phys. Lett. 2008, 93, 113103.

7. Li, X.; Cai, W.; An, J.; Kim, S.; Nah, J.; Yang, D.; Piner, R.; Velamakanni, A.; Jung, I.; Tutuc, E.; et al. Large-Area Synthesis of High-Quality and Uniform Graphene Films on Copper Foils. Science 2009, 324, 1312-1314.

8. López, G. The Solubility of C in Solid Cu. Scr. Mater. 2004, 51, 1-5.

9. McLellan, R. The Solubility of Carbon in Solid Gold, Copper, and Silver. Scr. Metall. 1969, 3, 389-391.

10. Oden, L. L.; Gokcen, N. A. Cu-C and Al-Cu-C Phase Diagrams and Thermodynamic Properties of $C$ in the Alloys from 1550 to $2300{ }^{\circ} \mathrm{C}$. Metall. Trans. B 1992, 23, 453-458.

11. Phase Diagrams of Binary Copper Alloys; Subramanian, P. R.; Chakrabati, D. J.; Laughlin, D. E., Eds.; ASM International: Materials Park, OH, 1994.

12. Li, X.; Cai, W.; Colombo, L.; Ruoff, R. S. Evolution of Graphene Growth on Ni and Cu by Carbon Isotope Labeling. Nano Lett. 2009, 9, 4268-4272.

13. Xu, Y.; Yan, X.-T. Chemical Vapour Deposition: An Integrated Engineering Design for Advanced Materials; Springer: New York, 2010.

14. Ohring, M. Materials Science of Thin Films, second ed.; Academic Press: San Diego, 2002.

15. Bhaviripudi, S.; Jia, X.; Dresselhaus, M. S.; Kong, J. Role of Kinetic Factors in Chemical Vapor Deposition Synthesis of Uniform Large Area Graphene Using Copper Catalyst. Nano Lett. 2010, 10, 4128-4133.

16. Luo, Z.; Kim, S.; Kawamoto, N.; Rappe, A. M.; Johnson, A. T. C. Growth Mechanism of Hexagonal-Shape Graphene Flakes with Zigzag Edges. ACS Nano 2011, 5, 9154-9160.

17. Colombo, L.; Li, X.; Han, B.; Magnuson, C.; Cai, W.; Zhu, Y.; Ruoff, R. S. Growth Kinetics and Defects of CVD Graphene on Cu. ECS Trans. 2010, 28, 109-114.

18. Zhang, W.; Wu, P.; Li, Z.; Yang, J. First-Principles Thermodynamics of Graphene Growth on Cu Surfaces. J. Phys. Chem. C 2011, 115, 17782-17787.

19. Kim, H.; Mattevi, C.; Calvo, M. R.; Oberg, J. C.; Artiglia, L.; Agnoli, S.; Hirjibehedin, C. F.; Chhowalla, M.; Saiz, E. 
Activation Energy Paths for Graphene Nucleation and Growth on Cu. ACS Nano 2012, 6, 3614-3623.

20. Loginova, E.; Bartelt, N. C.; Feibelman, P. J.; McCarty, K. F. Factors Influencing Graphene Growth on Metal Surfaces. New J. Phys. 2009, 11, 063046.

21. Shu, H.; Chen, X.; Tao, X.; Ding, F. Edge Structural Stability and Kinetics of Graphene Chemical Vapor Deposition Growth. ACS Nano 2012, 6, 3243-3250.

22. Mehdipour, H.; Ostrikov, K. K. Kinetics of Low-Pressure, LowTemperature Graphene Growth: Toward Single-Layer, SingleCrystalline Structure. ACS Nano 2012, 6, 10276-10286.

23. Li, Z.; Zhang, W.; Fan, X.; Wu, P.; Zeng, C.; Li, Z.; Zhai, X.; Yang, J.; Hou, J. Graphene Thickness Control via Gas-Phase Dynamics in Chemical Vapor Deposition. J. Phys. Chem. C 2012, 116, 10557-10562.

24. Davies, R.; Dinsdale, A.; Gisby, J.; Robinson, J.; Martin, S. MTDATA - Thermodynamic and Phase Equilibrium Software from the National Physical Laboratory. CALPHAD 2002, 26, 229-271.

25. Wu, B.; Geng, D.; Guo, Y.; Huang, L.; Xue, Y.; Zheng, J.; Chen, J.; Yu, G.; Liu, Y.; Jiang, L.; et al. Equiangular HexagonShape-Controlled Synthesis of Graphene on Copper Surface. Adv. Mater. 2011, 23, 3522-3525.

26. Yu, V.; Whiteway, E.; Maassen, J.; Hilke, M. Raman Spectroscopy of the Internal Strain of a Graphene Layer Grown on Copper Tuned by Chemical Vapor Deposition. Phys. Rev. B 2011, 205407.

27. Liu, L.; Zhou, H.; Cheng, R.; Chen, Y.; Lin, Y.-C.; Qu, Y.; Bai, J.; Ivanov, I. A.; Liu, G.; Huang, Y.; et al. A Systematic Study of Atmospheric Pressure Chemical Vapor Deposition Growth of Large-Area Monolayer Graphene. J. Mater. Chem. 2012, 22, 1498-1503.

28. Vlassiouk, I.; Smirnov, S.; Ivanov, I.; Fulvio, P. F.; Dai, S.; Meyer, H.; Chi, M.; Hensley, D.; Datskos, P.; Lavrik, N. V. Electrical and Thermal Conductivity of Low Temperature CVD Graphene: The Effect of Disorder. Nanotechnology 2011, 22, 275716

29. Celebi, K.; Cole, M. T.; Teo, K. B. K.; Park, H. G. Observations of Early Stage Graphene Growth on Copper. Electrochem. Solid State 2012, 15, K1-K4.

30. Zhang, B.; Lee, W. H.; Piner, R.; Kholmanov, I.; Wu, Y.; Li, H.; Ji, H.; Ruoff, R. S. Low Temperature Chemical Vapor Deposition Growth of Graphene from Toluene on Electropolished Copper Foils. ACS Nano 2012, 6, 2471-2476.

31. Li, X.; Magnuson, C. W.; Venugopal, A.; Tromp, R. M.; Hannon, J. B.; Vogel, E. M.; Colombo, L.; Ruoff, R. S. LargeArea Graphene Single Crystals Grown by Low-Pressure Chemical Vapor Deposition of Methane on Copper. J. Am. Chem. Soc. 2011, 133, 2816-2819.

32. Lee, S.; Lee, K.; Zhong, Z. Wafer Scale Homogeneous Bilayer Graphene Films by Chemical Vapor Deposition. Nano Lett. 2010, 10, 4702-4707.

33. Wu, W.; Yu, Q.; Peng, P.; Liu, Z.; Bao, J.; Pei, S.-S. Control of Thickness Uniformity and Grain Size in Graphene Films for Transparent Conductive Electrodes. Nanotechnology 2012, 23, 035603

34. Luo, Z.; Lu, Y.; Singer, D. W.; Berck, M. E.; Somers, L. A.; Goldsmith, B. R.; Johnson, A. T. C. Effect of Substrate Roughness and Feedstock Concentration on Growth of Wafer-Scale Graphene at Atmospheric Pressure. Chem. Mater. 2011, 23, 1441-1447.

35. Srivastava, A.; Galande, C.; Ci, L.; Song, L.; Rai, C.; Jariwala, D.; Kelly, K. F.; Ajayan, P. M. Novel Liquid Precursor-Based Facile Synthesis of Large-Area Continuous, Single, and FewLayer Graphene Films. Chem. Mater. 2010, 22, 3457-3461.

36. Vlassiouk, I.; Regmi, M.; Fulvio, P.; Dai, S.; Datskos, P.; Eres, G.; Smirnov, S. Role of Hydrogen in Chemical Vapor Deposition Growth of Large Single-Crystal Graphene. ACS Nano 2011, 5, 6069-6076.

37. Zhang, Y.; Li, Z.; Kim, P.; Zhang, L.; Zhou, C. Anisotropic Hydrogen Etching of Chemical Vapor Deposited Graphene. ACS Nano 2012, 6, 126-132.

38. Diaz-Pinto, C.; De, D.; Hadjiev, V. G.; Peng, H. AB-Stacked Multilayer Graphene Synthesized via Chemical Vapor
Deposition: A Characterization by Hot Carrier Transport. ACS Nano 2012, 6, 1142-1148.

39. Yao, Y.; Li, Z.; Lin, Z.; Moon, K.-S.; Agar, J.; Wong, C. Controlled Growth of Multilayer, Few-Layer, and SingleLayer Graphene on Metal Substrates. J. Phys. Chem. C 2011, 115, 5232-5238.

40. Lee, Y.; Bae, S.; Jang, H.; Jang, S.; Zhu, S.-E.; Sim, S. H.; Song, Y. I.; Hong, B. H.; Ahn, J.-H. Wafer-Scale Synthesis and Transfer of Graphene Films. Nano Lett. 2010, 10, 490-493.

41. Lenski, D. R.; Fuhrer, M. S. Raman and Optical Characterization of Multilayer Turbostratic Graphene Grown via Chemical Vapor Deposition. J. Appl. Phys. 2011, 110, 013720.

42. Han, G. H.; Güneș, F.; Bae, J. J.; Kim, E. S.; Chae, S. J.; Shin, H.-J.; Choi, J.-Y.; Pribat, D.; Lee, Y. H. Influence of Copper Morphology in Forming Nucleation Seeds for Graphene Growth. Nano Lett. 2011, 11, 4144-4148.

43. Bae, S.; Kim, H.; Lee, Y.; Xu, X.; Park, J.-S.; Zheng, Y.; Balakrishnan, J.; Lei, T.; Ri Kim, H.; Song, Y. I.; et al. Rollto-Roll Production of 30-in. Graphene Films for Transparent Electrodes. Nat. Nanotechnol. 2010, 5, 1-5.

44. Cao, H.; Yu, Q.; Jauregui, L. A.; Tian, J.; Wu, W.; Liu, Z.; Jalilian, R.; Benjamin, D. K.; Jiang, Z.; Bao, J.; et al. Electronic Transport in Chemical Vapor Deposited Graphene Synthesized on Cu: Quantum Hall Effect and Weak Localization. Appl. Phys. Lett. 2010, 96, 122106.

45. Hu, B.; Ago, H.; Ito, Y.; Kawahara, K.; Tsuji, M.; Magome, E.; Sumitani, K.; Mizuta, N.; Ikeda, K.; Mizuno, S. Epitaxial Growth of Large-Area Single-Layer Graphene over $\mathrm{Cu}(111)$ / Sapphire by Atmospheric Pressure CVD. Carbon 2012, 50, 57-65.

46. Huang, P. Y.; Ruiz-Vargas, C. S.; van der Zande, A. M.; Whitney, W. S.; Levendorf, M. P.; Kevek, J. W.; Garg, S.; Alden, J. S.; Hustedt, C. J.; Zhu, Y.; et al. Grains and Grain Boundaries in Single-Layer Graphene Atomic Patchwork Quilts. Nature 2011, 469, 389-392.

47. van der Zande, A. M.; Barton, R. A.; Alden, J. S.; Ruiz-Vargas, C. S.; Whitney, W. S.; Pham, P. H. Q.; Park, J.; Parpia, J. M.; Craighead, H. G.; McEuen, P. L. Large-Scale Arrays of SingleLayer Graphene Resonators. Nano Lett. 2010, 10, 48694873.

48. Li, X.; Magnuson, C. W.; Venugopal, A.; An, J.; Suk, J. W.; Han, B.; Borysiak, M.; Cai, W.; Velamakanni, A.; Zhu, Y.; et al. Graphene Films with Large Domain Size by a Two-Step Chemical Vapor Deposition Process. Nano Lett. 2010, 10, 4328-4334.

49. Rasool, H. I.; Song, E. B.; Mecklenburg, M.; Regan, B. C.; Wang, K. L.; Weiller, B. H.; Gimzewski, J. K. Atomic-Scale Characterization of Graphene Grown on Copper (100) Single Crystals. J. Am. Chem. Soc. 2011, 133, 12536-12543.

50. Robertson, A. W.; Warner, J. H. Hexagonal Single Crystal Domains of Few-Layer Graphene on Copper Foils. Nano Lett. 2011, 11, 1182-1189.

51. Rasool, H. I.; Song, E. B.; Allen, M. J.; Wassei, J. K.; Kaner, R. B.; Wang, K. L.; Weiller, B. H.; Gimzewski, J. K. Continuity of Graphene on Polycrystalline Copper. Nano Lett. 2011, 11, 251-256.

52. Wofford, J. M.; Nie, S.; McCarty, K. F.; Bartelt, N. C.; Dubon, O. D. Graphene Islands on Cu Foils: The Interplay between Shape, Orientation, and Defects. Nano Lett. 2010, 10, 4890-4896.

53. Cho, J.; Gao, L.; Tian, J.; Cao, H.; Wu, W.; Yu, Q.; Yitamben, E. N.; Fisher, B.; Guest, J. R.; Chen, Y. P.; et al. Atomic-Scale Investigation of Graphene Grown on Cu Foil and the Effects of Thermal Annealing. ACS Nano 2011, 5, 3607-3613.

54. Piekarczyk, W. Application of Thermodynamics to the Examination of the Diamond CVD Process from Hydrocarbon-Hydrogen Mixtures. J. Cryst. Growth 1989, 98, 765776.

55. Hüttinger, K. J. CVD in Hot Wall Reactors-The Interaction between Homogeneous Gas-Phase and Heterogeneous Surface Reactions. Chem. Vapor Depos. 1998, 04, 151-158.

56. Chen, H.; Zhu, W.; Zhang, Z. Contrasting Behavior of Carbon Nucleation in the Initial Stages of Graphene 
Epitaxial Growth on Stepped Metal Surfaces. Phys. Rev. Lett. 2010, 104, 186101.

57. Riikonen, S.; Krasheninnikov, A. V.; Halonen, L. O.; Nieminen, R. The Role of Stable and Mobile Carbon Adspecies in Copper-Promoted Graphene Growth.J. Phys. Chem. C 2012, 5802-5809.

58. Lin, Y.-Z.; Sun, J.; Yi, J.; Lin, J.-D.; Chen, H.-B.; Liao, D.-W. Energetics of Chemisorption and Conversion of Methane on Transition Metal Surfaces. J. Mol. Struct. (THEOCHEM) 2002, 587, 63-71.

59. Zhong, G.; Hofmann, S.; Yan, F.; Telg, H.; Warner, J. H.; Eder, D.; Thomsen, C.; Milne, W. I.; Robertson, J. Acetylene: A Key Growth Precursor for Single-Walled Carbon Nanotube Forests. J. Phys. Chem. C 2009, 113, 17321-17325.

60. Yuan, Q.; Gao, J.; Shu, H.; Zhao, J.; Chen, X.; Ding, F. Magic Carbon Clusters in the Chemical Vapor Deposition Growth of Graphene. J. Am. Chem. Soc. 2012, 134, 2970-2975.

61. Olsvik, O.; Rokstad, O. A.; Holmen, A. Pyrolysis of Methane in the Presence of Hydrogen. Chem. Eng. Technol. 1995, 18, 349-358.

62. Benzinger, W.; Becker, A.; Hüttinger, K. J. Chemistry and Kinetics of Chemical Vapour Deposition of Pyrocarbon: I. Fundamentals of Kinetics and Chemical Reaction Engineering. Carbon 1996, 34, 957-966.

63. Schwander, M.; Partes, K. A Review of Diamond Synthesis by CVD Processes. Diam. Relat. Mater. 2011, 20, 12871301.

64. Zhang, Y.; Zhang, L.; Kim, P.; Ge, M.; Li, Z.; Zhou, C. Vapor Trapping Growth of Single-Crystalline Graphene Flowers: Synthesis, Morphology, and Electronic Properties. Nano Lett. 2012, 12, 2810-2816.

65. Fan, L.; Zou, J.; Li, Z.; Li, X.; Wang, K.; Wei, J.; Zhong, M.; Wu, D.; Xu, Z.; Zhu, H. Topology Evolution of Graphene in Chemical Vapor Deposition, a Combined Theoretical/Experimental Approach toward Shape Control of Graphene Domains. Nanotechnology 2012, 23, 115605.

66. Murdock, A. T.; Koos, A.; Britton, T. B.; Houben, L.; Batten, T.; Zhang, T.; Wilkinson, A. J.; Dunin-Borkowski, R. E.; Lekka, C. E.; Grobert, N. Controlling the Orientation, Edge Geometry and Thickness of Chemical Vapor Depostion Graphene. ACS Nano 2013, 7, 1351-1359.

67. Keudell, A. von Formation of Polymer-Like Hydrocarbon Films from Radical Beams of Methyl and Atomic Hydrogen. Thin Solid Films 2002, 402, 1-37.

68. Werner, M.; Locher, R. Growth and Application of Undoped and Doped Diamond Films. Rep. Prog. Phys. 1998, 61, 1665-1710.

69. Wang, H.; Wang, G.; Bao, P.; Yang, S.; Zhu, W.; Xie, X.; Zhang, W.-J. Controllable Synthesis of Submillimeter Single-Crystal Monolayer Graphene Domains on Copper Foils by Suppressing Nucleation. J. Am. Chem. Soc. 2012, 134, 3627-3630.

70. Yu, Q.; Jauregui, L. A.; Wu, W.; Colby, R.; Tian, J.; Su, Z.; Cao, H.; Liu, Z.; Pandey, D.; Wei, D.; et al. Control and Characterization of Individual Grains and Grain Boundaries in Graphene Grown by Chemical Vapour Deposition. Nat. Mater. 2011, 10, 443-449.

71. Wu, Y. A.; Robertson, A. W.; Schäffel, F.; Speller, S. C.; Warner, J. H. Aligned Rectangular Few-Layer Graphene Domains on Copper Surfaces. Chem. Mater. 2011, 23, 4543-4547.

72. Pop, E.; Varshney, V.; Roy, A. K. Thermal Properties of Graphene: Fundamentals and Applications. MRS Bull. 2012, 37, 1273-1281. 